arXiv:1401.8195v3

\title{
Killing tensors and canonical geometry
}

\author{
M. Cariglia ${ }^{1 *}$ G. W.Gibbons ${ }^{2 \dagger}$, J.-W. van Holten ${ }^{3,4 \ddagger}$, \\ P. A. Horvathy ${ }^{5,6 \S}$, P. Kosiński ${ }^{7}$, P.-M. Zhang ${ }^{5,8 * *}$ \\ ${ }^{1}$ DEFIS, Universidade Federal de Ouro Preto, \\ Campus Moro de Cruzeiro, 35400-000 Ouro Preto, MG-Brasil \\ ${ }^{2}$ Department of Applied Mathematics and Theoretical Physics, \\ Cambridge University, Cambridge, UK \\ ${ }^{3}$ NIKHEF, Amsterdam (Netherlands) \\ 4 Leiden University, Leiden (Netherlands) \\ ${ }^{5}$ Institute of Modern Physics, Chinese Academy of Sciences, Lanzhou (China) \\ ${ }^{6}$ Laboratoire de Mathématiques et de Physique Théorique, Tours University (France) \\ ${ }^{7}$ Faculty of Physics and Applied Informatics, University of Lodz, (Poland) \\ 8 State Key Laboratory of Theoretical Physics, Institute of Theoretical Physics, \\ Chinese Academy of Sciences, Beijing 100190, China
}

(Dated: November 6, 2018)

\begin{abstract}
The systematic derivation of constants of the motion, based on Killing tensors and the gauge covariant approach, is outlined. Quantum dots are shown to support second-, third- and fourthrank Killing tensors.

KEY WORDS: Conserved quantities, Killing tensors, Covariant Dynamics, Quantum Dots

PACS numbers:

45.05. $+\mathrm{x}$, General theory of classical mechanics of discrete systems

11.30.Na, Nonlinear and dynamical symmetries (spectrum-generating symmetries)

73.21.La, Quantum dots
\end{abstract}

\footnotetext{
* e-mail:marco@iceb.ufop.br

$\dagger$ mail:G.W.Gibbons@damtp.cam.ac.uk

$\ddagger$ mail: t32@nikhef.nl

$\S$ e-mail:horvathy-at-lmpt.univ-tours.fr

ฯ email: pkosinsk@uni.lodz.pl

** e-mail:zhpm@impcas.ac.cn
} 


\section{Contents}

1. Introduction

2. Canonical approach to conservation laws

3. Application to Quantum Dots

4. Algebraic Structure

\section{Conclusion}

\section{Acknowledgments}

\section{References}

\section{INTRODUCTION}

Noether's theorem associates a conserved quantity to a symmetry, defined as a transformation of space-time which changes the Lagrangian by a total derivative [1]. Infinitesimally, such symmetries are Killing vectors. Higher-order expressions like the celebrated (Laplace-) Runge-Lenz vector cannot be obtained in this way, though, and require rather higher-rank generalizations called Killing tensors [2-7]. The relations between these concepts become particularly clear in the canonical formulation of the dynamics [8, 9].

The (Laplace-) Runge-Lenz vector is associated with rank-2 tensors. Killing tensors of rank $r \geq 2$ can also be considered, but physical examples are less common [10 15].

The main result of this paper is a systematic derivation of higher-rank conserved quantities based on Killing tensors, as illustrated by a fourth-rank conserved quantity, (3.24) below. It is obtained for Quantum Dots for a particular choice of the parameters, when the system is integrable but not separable [16-19]. Deriving this expression is far from being trivial : Blümel et al. [18], e.g., found the correct formula at their second attempt only (and gave no detailed explanation). The difficulty comes from that such higher-order expressions are, as said above, not associated with a simple geometric action on space-time and therefore cannot be derived by the original Noether theorem. 


\section{CANONICAL APPROACH TO CONSERVATION LAWS}

For particles coupled to scalar and vector potentials $\left(\Phi, A_{i}\right)$ the hamiltonian takes the form

$$
H=\frac{1}{2} g^{i j}(q) \Pi_{i} \Pi_{j}+\Phi(q)
$$

where the $\Pi_{i}=p_{i}-e A_{i}$ denote the gauge-covariant momenta; $e$ is the charge. The covariant brackets read [8, 9]

$$
\{Q, K\}=D_{i} Q \frac{\partial K}{\partial \Pi_{i}}-\frac{\partial Q}{\partial \Pi_{i}} D_{i} K+e F_{i j}(q) \frac{\partial Q}{\partial \Pi_{i}} \frac{\partial K}{\partial \Pi_{j}},
$$

where $F_{i j}=\partial_{i} A_{j}-\partial_{j} A_{i}$ is the field-strength tensor of $A_{i}$ on the configuration space with metric $g_{i j}$, and the covariant derivatives are defined by

$$
\left.D_{i} K \equiv \frac{\partial K}{\partial q^{i}}\right|_{\Pi}+\Gamma_{i j}^{k} \Pi_{k} \frac{\partial K}{\partial \Pi_{j}} .
$$

The definition reproduces the canonical equations of motion:

$$
\begin{gathered}
\frac{d q^{i}}{d t}=\left\{q^{i}, H\right\}=\frac{\partial H}{\partial \Pi_{i}} \Leftrightarrow \quad \Pi_{i}=g_{i j} \frac{d q^{j}}{d t}, \\
\frac{d \Pi_{i}}{d t}=\left\{\Pi_{i}, H\right\}=-D_{i} H+e F_{i j} \frac{\partial H}{\partial \Pi_{j}} \\
\Leftrightarrow \quad \frac{D \Pi_{i}}{D t} \equiv \frac{d \Pi_{i}}{d t}-\frac{d q^{j}}{d t} \Gamma_{j i}{ }^{k} \Pi_{k}=e F_{i}{ }^{j} \Pi_{j}-\frac{\partial \Phi}{\partial q^{i}} .
\end{gathered}
$$

Constants of the motion which are polynomials in the covariant momenta are obtained by solving eq.

$$
\{Q, H\}=0 \quad \text { with } \quad Q=\sum_{n \geq 0} \frac{1}{n !} C^{(n) i_{1} \ldots i_{n}}(q) \Pi_{i_{1}} \ldots \Pi_{i_{n}},
$$

which leads to the generalized Killing equations

$$
\begin{array}{ll}
C^{(1) i} \Phi_{; i}=0, \quad C_{; i}^{(0)}=e C^{(1) j} F_{i j}+C_{i}^{(2) j} \Phi_{; j}, & \\
C_{\left(i_{1} . . i_{n} ; i_{n+1}\right)}^{(n)}=e C_{\left(i_{1} \ldots i_{n}\right.}^{(n+1) j} F_{\left.i_{n+1}\right) j}+C_{i_{1} \ldots i_{n+1}}^{(n+2) j} \Phi_{; j}, \quad n \geq 1,
\end{array}
$$

as discussed in Ref. [9]. Note that any $Q$ which is a polynomial in $\Pi_{i}$ of rank $n$, has $C^{(m)}=0$ for all $m>n$. In that case the highest coefficient tensor $C^{(n)}$ is a again a rank- $n$ Killing tensor:

$$
C_{\left(i_{1} \ldots i_{n} ; i_{n+1}\right)}^{(n)}=0
$$

the next-to-highest coefficient tensor satisfies

$$
C_{\left(i_{1} \ldots i_{n-1} ; i_{n}\right)}^{(n-1)}=e C_{\left(i_{1} \ldots i_{n-1}\right.}^{(n) j} F_{\left.i_{n}\right) j}
$$


and all tensors of rank $n-2$ and lower are subject to the full equation (2.7). Furthermore, the generalized Killing vector $C^{(1)}$ is always required to be orthogonal to the gradient of the scalar potential.

\section{APPLICATION TO QUANTUM DOTS}

The above formalism can be applied to the Quantum-Dot model of ref. [16-19]. This concerns two charged particles with Coulomb interaction in a constant magnetic field and a confining oscillator potential. The hamiltonian is

$$
H=\sum_{a=1}^{2}\left[\frac{1}{2} \boldsymbol{\Pi}_{a}^{2}+U\left(\mathbf{r}_{a}\right)\right]-\frac{a}{\left|\mathbf{r}_{1}-\mathbf{r}_{2}\right|} .
$$

The magnetic field direction is the $z$-direction, and the confining oscillator potential is taken to be axially symmetric:

$$
U\left(\mathbf{r}_{a}\right)=\frac{1}{2}\left[\omega_{0}^{2}\left(x_{a}^{2}+y_{a}^{2}\right)+\omega_{z}^{2} z_{a}^{2}\right] .
$$

Transformation to center-of-mass co-ordinates $\mathbf{r}_{1,2}=\frac{1}{\sqrt{2}}(\mathbf{R} \pm \mathbf{r}), \boldsymbol{\Pi}_{1,2}=\frac{1}{\sqrt{2}}(\boldsymbol{\Pi} \pm \boldsymbol{\pi})$, leads to separation of variables, $H\left(\mathbf{r}_{a}, \boldsymbol{\Pi} i_{a}\right)=H_{C M}(\mathbf{R}, \boldsymbol{\Pi})+H_{r e d}(\mathbf{r}, \boldsymbol{\pi})$, with $H_{C M}=\frac{1}{2} \boldsymbol{\Pi}^{2}+U(\mathbf{R})$ and

$$
H_{\text {red }}=\frac{1}{2} \boldsymbol{\pi}^{2}+U(\mathbf{r})-\frac{a}{r} .
$$

As the magnetic field is constant, $F_{x y}=-F_{y x}=B, F_{y z}=F_{z x}=0$, the covariant brackets separate as well: $\{Q, K\}=\{Q, K\}_{C M}+\{Q, K\}_{\text {red }}$. In euclidean co-ordinates they read

$$
\begin{gathered}
\{K, Q\}_{C M}=\frac{\partial K}{\partial R^{i}} \frac{\partial Q}{\partial \Pi_{i}}-\frac{\partial K}{\partial \Pi_{i}} \frac{\partial Q}{\partial R^{i}}+e B\left(\frac{\partial K}{\partial \Pi_{x}} \frac{\partial Q}{\partial \Pi_{y}}-\frac{\partial K}{\partial \Pi_{y}} \frac{\partial K}{\partial \Pi_{x}}\right) \\
\{K, Q\}_{r e d}=\frac{\partial K}{\partial r^{i}} \frac{\partial Q}{\partial \pi_{i}}-\frac{\partial K}{\partial \pi_{i}} \frac{\partial Q}{\partial r^{i}}+e B\left(\frac{\partial K}{\partial \pi_{x}} \frac{\partial Q}{\partial \pi_{y}}-\frac{\partial K}{\partial \pi_{y}} \frac{\partial K}{\partial \pi_{x}}\right) .
\end{gathered}
$$

In the following we restrict ourselves to the effective 1-particle problem defined by the reduced brackets and hamiltonian $H_{\text {red }}$. To apply the formalism of generalized Killing equations we lump the oscillator and Coulomb potential into the single scalar potential

$$
\Phi=\frac{1}{2}\left[\omega_{0}^{2}\left(x^{2}+y^{2}\right)+\omega_{z}^{2} z^{2}\right]-\frac{a}{\sqrt{x^{2}+y^{2}+z^{2}}} .
$$

The equations of motion in $3 \mathrm{D}$ euclidean co-ordinates derived from the reduced hamiltonian and brackets then read

$$
\dot{r}_{i}=\left\{r_{i}, H\right\}=\pi_{i}, \quad \dot{\pi}_{i}=\left\{\pi_{i}, H\right\}=-\Phi_{, i}+\epsilon_{i j z} e B \pi_{j}
$$


where the comma denotes an ordinary partial derivative w.r.t. $r_{i}$, and $\epsilon_{i j k}$ is the permutation tensor. The corresponding quantum theory is obtained by replacing the brackets of phasespace functions $(K, Q)$ by operator commutation relations.

To make use of axial symmetry, it is convenient to transform to curvilinear co-ordinates $\xi^{i}=(\rho, z, \varphi)$. Then the hamiltonian becomes

$$
H=\frac{1}{2} g^{i j} \pi_{i} \pi_{j}+\Phi,
$$

with metric $g_{i j}=\operatorname{diag}\left(1,1, \rho^{2}\right)$ and scalar potential (3.5). As $F_{\rho \varphi}=\rho B$, the covariant brackets are given by

$$
\{K, Q\}=D_{i} K \frac{\partial Q}{\partial \pi_{i}}-\frac{\partial K}{\partial \pi_{i}} D_{i} Q+e B \rho\left(\frac{\partial K}{\partial \pi_{\rho}} \frac{\partial Q}{\partial \pi_{\varphi}}-\frac{\partial K}{\partial \pi_{\varphi}} \frac{\partial Q}{\partial \pi_{\rho}}\right),
$$

where the covariant derivatives $D_{i}$ are defined by (2.3) with non-zero connection coefficients $\Gamma_{\rho \varphi}^{\varphi}=1 / \rho, \Gamma_{\varphi \varphi}^{\rho}=-\rho$. As the angle $\varphi$ is a cyclic co-ordinate, the corresponding canonical momentum is conserved. In the present covariant formalism this follows from the existence of a Killing vector and a Killing scalar, $\left(C^{(1) \rho}, C^{(1) z}, C^{(1) \varphi}\right)=(0,0,1), C^{(0)}=\omega_{L} \rho^{2}$, respectively, where $\omega_{L}=e B / 2$. They combine into the constant of the motion, namely the $z$-component of the total angular momentum,

$$
L_{z}=C^{(1) i} \pi_{i}+C^{(0)}=\pi_{\varphi}+\omega_{L} \rho^{2} .
$$

The Hamilton equations $d \xi^{i} / d t=\left\{\xi^{i}, H\right\}=g^{i j} \pi_{j}$ imply $d \varphi / d t=\pi_{\varphi} / \rho^{2}=L_{z} / \rho^{2}-\omega_{L} ; \omega_{L}$ is hence the Larmor frequency.

As discussed in [16 19], the model allows for more constants of motion whenever certain specific conditions on the frequencies hold, namely for certain exceptional values of $\tau=$ $\omega_{z} / \sqrt{\omega_{0}^{2}+\omega_{L}^{2}}$.

- We first observe that there is a rank-2 Killing tensor

$$
C_{i j}^{(2)}=\left(\begin{array}{ccc}
2 z & -\rho & 0 \\
-\rho & 0 & 0 \\
0 & 0 & 2 \rho^{2} z
\end{array}\right) \Leftrightarrow \frac{1}{2} C^{(2) i j} \pi_{i} \pi_{j}=z \pi_{\rho}^{2}-\rho \pi_{\rho} \pi_{z}+\frac{z}{\rho^{2}} \pi_{\varphi}^{2} .
$$

Then the generalized Killing equation (2.9) for $C^{(1)}$ is solved by

$$
C_{i}^{(1)}=\left(0,0, e B \rho^{2} z\right) \quad \Leftrightarrow \quad C^{(1) i} \pi_{i}=e B z \pi_{\varphi} .
$$


This vector is orthogonal to the gradient of $\Phi$, as required by the first equation in (2.7). Finally, the Killing scalar $C^{(0)}$ must satisfy

$$
\begin{aligned}
& C_{, \rho}^{(0)}=\left(4 \omega_{L}^{2}+2 \omega_{0}^{2}-\omega_{z}^{2}\right) \rho z+\frac{a \rho z}{\left(\rho^{2}+z^{2}\right)^{3 / 2}}, \\
& C_{, z}^{(0)}=-\omega_{\rho}^{2} \rho^{2}-\frac{a \rho^{2}}{\left(\rho^{2}+z^{2}\right)^{3 / 2}}, \quad C_{, \varphi}^{(0)}=0 .
\end{aligned}
$$

A solution, namely

$$
C^{(0)}=-\omega_{\rho}^{2} \rho^{2} z-\frac{a z}{\sqrt{\rho^{2}+z^{2}}},
$$

exists, provided the frequencies satisfy

$$
\omega_{0}^{2}+\omega_{L}^{2}=\frac{1}{4} \omega_{z}^{2}
$$

Combining the results, the full constant of motion,

$$
Q_{1}=z \pi_{\rho}^{2}-\rho \pi_{\rho} \pi_{z}+\frac{z}{\rho^{2}} \pi_{\varphi}^{2}+2 \omega_{L} z \pi_{\varphi}-\omega_{0}^{2} \rho^{2} z-\frac{a z}{\sqrt{\rho^{2}+z^{2}}},
$$

We recover hence the Runge-Lenz-type quadratic expression found before for $\tau=2$, when the system is separable in parabolic coordinates [16 19].

Another constant can be constructed starting from the rank-4 Killing tensor

$$
\frac{1}{4 !} C^{(4) i j k l} \pi_{i} \pi_{j} \pi_{k} \pi_{l}=\rho^{2} \pi_{z}^{4}-2 \rho z \pi_{\rho} \pi_{z}^{3}+z^{2} \pi_{\rho}^{2} \pi_{z}^{2}+\frac{1}{\rho^{2}} \pi_{\varphi}^{4}+\pi_{\rho}^{2} \pi_{\varphi}^{2}+\left(2+\frac{z^{2}}{\rho^{2}}\right) \pi_{z}^{2} \pi_{\varphi}^{2} .
$$

Then solving eqs. (2.9) for $C^{(3)}$ one finds the minimal solution

$$
\frac{1}{3 !} C^{(3) i j k} \pi_{i} \pi_{j} \pi_{k}=2 \omega_{L} \pi_{\varphi}\left(\rho^{2} \pi_{\rho}^{2}+\left(2 \rho^{2}+z^{2}\right) \pi_{z}^{2}\right)
$$

modulo a rather long list of separate rank-3 Killing tensor expressions, defining independent constants of motion. These are discussed in eq. (3.25) below.

The terms quadratic in the covariant momenta are now obtained in a straightforward way by requiring all contributions of order $\pi^{3}$ to the bracket $\{Q, H\}$ to cancel; this gives the minimal expression,

$$
\begin{aligned}
\frac{1}{2} C^{(2) i j} \pi_{i} \pi_{j}= & {\left[\left(2 \omega_{z}^{2}-\omega_{0}^{2}\right) z^{2} \rho^{2}+2 \omega_{L}^{2} \rho^{4}-\frac{2 a \rho^{2}}{\sqrt{z^{2}+\rho^{2}}}\right] \pi_{z}^{2} } \\
& +\left[\frac{2}{3}\left(2 \omega_{0}^{2}-5 \omega_{z}^{2}+2 \omega_{L}^{2}\right) z^{3} \rho+\frac{2 a z \rho}{\sqrt{z^{2}+\rho^{2}}}\right] \pi_{z} \pi_{\rho} \\
& +\left[\omega_{L}^{2} \rho^{4}-\frac{1}{3}\left(\omega_{0}^{2}-4 \omega_{z}^{2}+\omega_{L}^{2}\right) z^{4}\right] \pi_{\rho}^{2} \\
& +\left[2 \omega_{z}^{2} z^{2}+\left(\omega_{0}^{2}-5 \omega_{L}^{2}\right) \rho^{2}-\frac{1}{3}\left(\omega_{0}^{2}-4 \omega_{z}^{2}+\omega_{L}^{2}\right) \frac{z^{4}}{\rho^{2}}-\frac{2 a}{\sqrt{z^{2}+\rho^{2}}}\right] \pi_{\varphi}^{2}
\end{aligned}
$$


Calculating the contributions of order $\pi^{2}$, we have to add a linear term

$$
C^{(1) i} \pi_{i}=-2 \omega_{L} \pi_{\varphi}\left[\frac{1}{3}\left(\omega_{0}^{2}-4 \omega_{z}^{2}+\omega_{L}^{2}\right) z^{4}-2 \omega_{z}^{2} z^{2} \rho^{2}+\left(3 \omega_{L}^{2}-\omega_{0}^{2}\right) \rho^{4}+\frac{2 a \rho^{2}}{\sqrt{z^{2}+\rho^{2}}}\right] .
$$

It remains to find a $C^{(0)}(z, \rho)$ such that the bracket closes. This requires

$$
\begin{aligned}
C_{, z}^{(0)}= & \left(4 \omega_{z}^{4}-\frac{16}{3} \omega_{z}^{2} \omega_{\rho}^{2}+\frac{4}{3} \omega_{0}^{4}+\frac{4}{3} \omega_{L}^{2} \omega_{0}^{2}\right) z^{3} \rho^{2}+4 \omega_{L}^{2} \omega_{z}^{2} z \rho^{4}-\frac{2 a^{2} z \rho^{2}}{\left(z^{2}+\rho^{2}\right)^{2}} \\
& +\frac{a}{\left(z^{2}+\rho^{2}\right)^{3 / 2}}\left[\left(-\frac{10}{3} \omega_{z}^{2}+\frac{4}{3} \omega_{0}^{2}+\frac{4}{3} \omega_{L}^{2}\right) z^{3} \rho^{2}+\left(-4 \omega_{z}^{2}+2 \omega_{0}^{2}+4 \omega_{L}^{2}\right) z \rho^{4}\right] \\
C_{, \rho}^{(0)}= & \left(-\frac{10}{3} \omega_{z}^{4}+4 \omega_{z}^{2} \omega_{0}^{2}-\frac{2}{3} \omega_{0}^{4}+\frac{20}{3} \omega_{L}^{2} \omega_{z}^{2}-2 \omega_{L}^{2} \omega_{0}^{2}-\frac{4}{3} \omega_{L}^{4}\right) z^{4} \rho \\
& +8 \omega_{L}^{2} \omega_{z}^{2} z^{2} \rho^{3}-6 \omega_{L}^{2}\left(2 \omega_{L}^{2}-\omega_{0}^{2}\right) \rho^{5}+\frac{2 a^{2} z^{2} \rho}{\left(z^{2}+\rho^{2}\right)^{2}} \\
& +\frac{a}{\left(z^{2}+\rho^{2}\right)^{3 / 2}}\left[\frac{2}{3}\left(\omega_{0}^{2}+2 \omega_{z}^{2}+\omega_{L}^{2}\right) z^{4} \rho+2\left(\omega_{z}^{2}-4 \omega_{L}^{2}\right) z^{2} \rho^{3}-6 \omega_{L}^{2} \rho^{5}\right]
\end{aligned}
$$

Let us first turn off the Coulomb potential, i.e., consider the $a$-independent part of these eqns. Then the integrability condition is

$$
\left(2 \omega_{L}^{2}-5 \omega_{z}^{2}+2 \omega_{0}^{2}\right)^{2}=9 \omega_{z}^{4}
$$

with allows for two solutions, namely

$$
\text { (a) } \omega_{L}^{2}=4 \omega_{z}^{2}-\omega_{0}^{2}, \quad \text { (b) } \omega_{L}^{2}=\omega_{z}^{2}-\omega_{0}^{2} \text {. }
$$

Thus, for the magnetic problem with a confining harmonic potential but with no Coulomb potential, there are two values of the Larmor frequency $\omega_{L}$ for which there is a quartic constant of motion.

- In contrast, when $a \neq 0$, i.e., when the Coulomb potential is switched on, the terms linear in $a$ are integrable only if condition (a) is satisfied (terms proportional to $a^{2}$ are actually always integrable) - which is, indeed, the integrable but non-separable case $\tau=1 / 2$ in [19] cf. [16 19]. Imposing condition (a), the minimal solution for $C^{(0)}$ becomes

$$
\begin{aligned}
C^{(0)}= & \omega_{z}^{4} z^{4} \rho^{2}+2 \omega_{z}^{2} \omega_{L}^{2} z^{2} \rho^{4}-\omega_{L}^{2}\left(3 \omega_{L}^{2}-4 \omega_{z}^{2}\right) \rho^{6} \\
& +\frac{2 a}{\sqrt{z^{2}+\rho^{2}}}\left[\omega_{z}^{2} z^{2} \rho^{2}-\omega_{L}^{2} \rho^{4}\right]-\frac{a^{2}}{2} \frac{z^{2}-\rho^{2}}{z^{2}+\rho^{2}},
\end{aligned}
$$


with $\omega_{L}^{2}+\omega_{0}^{2}=4 \omega_{z}^{2}$. Then the sum of the expressions (3.16), (3.17), (3.18), (3.19) and (3.23) represents, for this special value of the magnetic field, the quartic constant of the motion in the CM system. Explicitly,

$$
\begin{aligned}
Q_{2}= & \rho^{2} \pi_{z}^{4}-2 \rho z \pi_{\rho} \pi_{z}^{3}+z^{2} \pi_{\rho}^{2} \pi_{z}^{2}+\frac{1}{\rho^{2}} \pi_{\varphi}^{4}+\pi_{\rho}^{2} \pi_{\varphi}^{2}+\left(2+\frac{z^{2}}{\rho^{2}}\right) \pi_{z}^{2} \pi_{\varphi}^{2} \\
& +2 \omega_{L} \pi_{\varphi}\left(\rho^{2} \pi_{\rho}^{2}+\left(2 \rho^{2}+z^{2}\right) \pi_{z}^{2}\right)+\left[\left(2 \omega_{z}^{2}-\omega_{0}^{2}\right) z^{2} \rho^{2}+2 \omega_{L}^{2} \rho^{4}-\frac{2 a \rho^{2}}{\sqrt{z^{2}+\rho^{2}}}\right] \pi_{z}^{2} \\
& +\left[\frac{2}{3}\left(2 \omega_{0}^{2}-5 \omega_{z}^{2}+2 \omega_{L}^{2}\right) z^{3} \rho+\frac{2 a z \rho}{\sqrt{z^{2}+\rho^{2}}}\right] \pi_{z} \pi_{\rho}+\left[\omega_{L}^{2} \rho^{4}-\frac{1}{3}\left(\omega_{0}^{2}-4 \omega_{z}^{2}+\omega_{L}^{2}\right) z^{4}\right] \pi_{\rho}^{2} \\
& +\left[2 \omega_{z}^{2} z^{2}+\left(\omega_{0}^{2}-5 \omega_{L}^{2}\right) \rho^{2}-\frac{1}{3}\left(\omega_{0}^{2}-4 \omega_{z}^{2}+\omega_{L}^{2}\right) \frac{z^{4}}{\rho^{2}}-\frac{2 a}{\sqrt{z^{2}+\rho^{2}}}\right] \pi_{\varphi}^{2} \\
& -2 \omega_{L} \pi_{\varphi}\left[\frac{1}{3}\left(\omega_{0}^{2}-4 \omega_{z}^{2}+\omega_{L}^{2}\right) z^{4}-2 \omega_{z}^{2} z^{2} \rho^{2}+\left(3 \omega_{L}^{2}-\omega_{0}^{2}\right) \rho^{4}+\frac{2 a \rho^{2}}{\sqrt{z^{2}+\rho^{2}}}\right] \\
& +\omega_{z}^{4} z^{4} \rho^{2}+2 \omega_{z}^{2} \omega_{L}^{2} z^{2} \rho^{4}-\omega_{L}^{2}\left(3 \omega_{L}^{2}-4 \omega_{z}^{2}\right) \rho^{6}+\frac{2 a}{\sqrt{z^{2}+\rho^{2}}}\left[\omega_{z}^{2} z^{2} \rho^{2}-\omega_{L}^{2} \rho^{4}\right]-\frac{a^{2}}{2} \frac{z^{2}-\rho^{2}}{z^{2}+\rho^{2}},
\end{aligned}
$$

which is in fact the conserved quantity found in the integrable-but-non-separable case $\tau=$ 1/2 using quite different methods [19]. By construction, the coefficients of the quartic term (3.16) define a rank-4 Killing tensor w.r.t. the metric (3.7).

More generally, the complete list of rank-3 Killing tensors is

$$
\begin{array}{rlrl}
K_{1}^{(3)} & =\pi_{z}^{3}, & K_{2}^{(3)} & =\pi_{\varphi}^{3} \\
K_{3}^{(3)} & =\pi_{z} \pi_{\varphi}^{2}, & K_{4}^{(3)} & =\pi_{z}\left(\pi_{\rho}^{2}+\frac{1}{\rho^{2}} \pi_{\varphi}^{2}\right) \\
K_{5}^{(3)} & =\pi_{\varphi}\left(\pi_{\rho}^{2}+\frac{1}{\rho^{2}} \pi_{\varphi}^{2}\right), & K_{6}^{(3)} & =\pi_{z}\left[-\rho \pi_{\rho} \pi_{z}+z\left(\pi_{\rho}^{2}+\frac{1}{\rho^{2}} \pi_{\varphi}^{2}\right)\right], \\
K_{7}^{(3)} & =\pi_{z}\left[\frac{1}{2} \rho^{2} \pi_{z}^{2}-z \rho \pi_{\rho} \pi_{z}+\frac{1}{2} z^{2}\left(\pi_{\rho}^{2}+\frac{1}{\rho^{2}} \pi_{\varphi}^{2}\right)\right] .
\end{array}
$$

Note, however, that these are composed of direct products of lower-rank Killing tensors and vectors. In particular, $K_{z}^{(1)}=\pi_{z}$ and $K_{\varphi}^{(1)}=\pi_{\varphi}$ define Killing vectors by themselves: $K_{z}^{(1) i}=(0,1,0), K_{\varphi}^{(1) i}=(0,0,1)$.

The expressions in eqs. (3.25) are products of these Killing vectors with each other and with the rank-2 Killing tensors

$$
\begin{aligned}
& K_{1}^{(2)}=\pi_{\rho}^{2}+\frac{1}{\rho^{2}} \pi_{\varphi}^{2}, \quad K_{2}^{(2)}=-\rho \pi_{\rho} \pi_{z}+z\left(\pi_{\rho}^{2}+\frac{1}{\rho^{2}} \pi_{\varphi}^{2}\right), \\
& K_{3}^{(2)}=\frac{1}{2} \rho^{2} \pi_{z}^{2}-z \rho \pi_{\rho} \pi_{z}+\frac{1}{2} z^{2}\left(\pi_{\rho}^{2}+\frac{1}{\rho^{2}} \pi_{\varphi}^{2}\right) .
\end{aligned}
$$


We discuss these expressions in turn. First, $K_{1}^{(2)}$ satisfies the bracket relation

$$
\left\{K_{1}^{(2)}, H\right\}=-2 \rho \pi_{\rho}\left(\omega_{0}^{2}+\frac{a}{\left(z^{2}+\rho^{2}\right)^{3 / 2}}\right) .
$$

Now as $\pi_{\rho}$ is not a Killing vector, $K_{1}^{(2)}$ can be turned into a constant of motion only by adding a scalar term $K_{1}^{(0)}$ such that

$$
K_{1, z}^{(0)}=0, \quad K_{1, \rho}^{(0)}=2 \rho\left(\omega_{0}^{2}+\frac{a}{\left(z^{2}+\rho^{2}\right)^{3 / 2}}\right) .
$$

The solution of the 2 nd equation:

$$
K_{1}^{(0)}=\omega_{0}^{2} \rho^{2}-\frac{a}{\sqrt{z^{2}+\rho^{2}}}
$$

satisfies the first equation (3.28) only if $a=0$. Therefore this quadratic Killing tensor generates a constant of motion only in the absence of a Coulomb potential: $a=0$.

An alternative is to replace the $3 \mathrm{D}$ Coulomb potential by a $2 \mathrm{D}$ one:

$$
\Phi \rightarrow \tilde{\Phi}=\frac{1}{2} \omega_{z}^{2} z^{2}+\frac{1}{2} \omega_{0}^{2} \rho^{2}-\frac{a}{\rho} .
$$

Next, observe that $K_{2}^{(2)}$ is identical to the Killing tensor (3.10). We have already seen that it can be extended to a constant of motion only if the Larmor frequency is tuned to take the value (3.14).

Finally, we discuss $K_{3}^{(2)}$. A straightforward calculation along the previous lines shows that it can also be extended to a complete constant of motion,

$$
Q_{3}=\frac{1}{2} \rho^{2} \pi_{z}^{2}-z \rho \pi_{z} \pi_{\rho}+\frac{1}{2} z^{2}\left(\pi_{\rho}^{2}+\frac{1}{\rho^{2}} \pi_{\varphi}^{2}\right)+\omega_{L} z^{2} \pi_{\varphi}+\frac{1}{2} \omega_{L}^{2} z^{2} \rho^{2},
$$

provided $\omega_{L}^{2}=\omega_{z}^{2}-\omega_{0}^{2}$, - which is condition (b) in (3.22).

We observe that (3.31) is in fact the difference of two separately conserved quantities found in Ref. [19], $\frac{1}{2}\left(L^{2}-L_{z}^{2}\right)$, i.e., (half of) the total angular momentum squared, $L^{2}$, minus $L_{z}^{2}$, the square of the third component of $L_{z}$. This is no surprise since condition (b) in (3.22) means $\tau=1$, which amounts to spherical symmetry and hence conserved total angular momentum after elimination of the magnetic field [19]. 


\section{ALGEBRAIC STRUCTURE}

When we have several symmetries, their algebraic structure is of fundamental importance. (Remember the o(4)/o(3,1) dynamical symmetry of the Kepler problem.) How do the Killing tensors reflect the Poisson algebra structure of the associated conserved quantities?

The answer is non-trivial let alone in the simplest, rank-1 case, when the Poisson structure of the conserved quantities may not be the same as that of the generating vectors under Lie bracket. Just consider a free particle: while the vectors of the infinitesimal action on spacetime span the center-less Galilei Lie algebra, the associated conserved quantities realize its central extension (called the Bargmann algebra).

This problem can be conveniently dealt with using a higher-dimensional framework [20] and Schouten-Nijenhuis algebras [12]. Full details will be presented elsewhere. Here we satisfy ourselves with some general remarks about the bracket algebra [21]. Let $J^{(p)}$ be constructed from a highest-rank Killing tensor of rank p. By construction, the bracket of two such constants of motion has the general structure

$$
\left\{J^{(p)}, J^{(q)}\right\} \sim J^{(p+q-1)}
$$

It follows that the generators of rank $p=1$ form a closed algebra, namely a Lie algebra if the structure functions are constant. The constants of rank $p \geq 1$ then must form representations of this algebra:

$$
\left\{J^{(p)}, J^{(1)}\right\} \sim J^{(p)}
$$

If there is more than one constant of the motion of rank $p, q \geq 2$, their bracket generates

constants of motion of higher rank $p+q-1 \geq p+1$. Therefore either the $J^{(p)}, p \geq 2$, form a trivial representation of the Lie algebra and all their brackets vanish, or an infinitedimensional set of constants of the motion is generated. Well-known examples of such infinite-dimensional algebras are the Virasoro and Kac-Moody algebras. However, these non-trivial infinite-dimensional algebras arise only for infinite-dimensional systems. In the finite-dimensional case we expect the brackets of higher-rank constants of motion to vanish, or represent simple powers and products of lower-rank constants.

Obviously, the Lie-algebra of constants $J^{(1)}$ generates transformations in configuration space, and corresponding configuration-dependent linear transformations in momentum 
space separately:

$$
J^{(1)}=\xi^{i}(q) p_{i} \quad \Rightarrow \quad\left\{q^{i}, J^{(i)}\right\}=\xi^{i}(q), \quad\left\{q^{i}, J^{(i)}\right\}=-\partial_{i} \xi^{j}(q) p_{j}
$$

In contrast, the higher-rank constants generate transformations in phase space, which mix configuration- and momentum variables $\left(q^{i}, p^{i}\right)$ - just like in the Kepler case.

\section{CONCLUSION}

To summarize our results, we derived, after outlining a general covariant framework based on higher-rank Killing tensors, three constants of the motion for the Quantum Dot Hamiltonian (3.7) with trapping potential (3.5). These additional conserved quantities arise for specific values of the magnetic field strength $B$. Note that terms which are odd in the momenta are multiplied by odd powers of the Larmor frequency consistently with timereversal symmetry.

Case (3.14) i.e. $\omega_{L}^{2}=\frac{1}{4} \omega_{z}^{2}-\omega_{0}^{2}$ corresponds to $\tau=2$ which is the one separable in parabolic coordinates and admits the quadratic "Runge-Lenz-type scalar" constant of motion (3.15).

For Case (a) in (3.22) i.e., for $\omega_{L}^{2}=4 \omega_{z}^{2}-\omega_{0}^{2}$, there is a quartic constant of the motion, namely $Q_{2}$ (3.24), consistent with the expression found in Ref. [19] in the integrable but non-separable case $\tau=1 / 2$.

In Case (b) in ( $(\underline{3.22})$, i.e., for $\omega_{L}^{2}=\omega_{z}^{2}-\omega_{0}^{2}$, we have another quadratic constant of motion, namely $Q_{3}$ in (3.31), which is in fact the difference of the total angular momentum squared minus the square of the third component, found in Ref. [19] for $\tau=1$ i.e., when the system is hiddenly and effectively spherically symmetric.

In the the integrable cases $\tau=1,2,1 / 2$ we have found a maximal set of mutually commuting conserved quantities. No other independent and mutually commuting quantities can be found. However, this does not exclude a priori the possibility that the system is super-integrable and that further conserved quantities exist, that would not commute with the ones we had found. Our systematic analysis implies that any such further conserved quantity, if it does exist, must be either of order $>4$ or non-polynomial in the momenta.

At last, one might wonder if one could not start with a systematic determination of all Killing tensors. This should in principle be possible by solving the Killing equations for any given rank, generalizing the standard procedure followed for Killing vectors. This is a rather 
laborious task, though, and the only results we are aware of concern rank-two tensors in the free case, with a huge number of Killing tensors [22].

More details and examples are discussed in a follow-up paper [23].

\section{Acknowledgments}

For $\mathrm{JWvH}$ this work is part of the research program of the Foundation for Research of Matter (FOM). P.K. would like to acknowledge Pawel Maslanka and Cezary Gonera for discussions. This work was partially supported also by the National Natural Science Foundation of China (Grants No. 11035006 and 11175215) and by the Chinese Academy of Sciences Visiting Professorship for Senior International Scientists (Grant No. 2010TIJ06).

[1] E. Noether, "Invariante Variationsprobleme," Nachr. Königl. Gesellsch. Wiss. zu Göttingen (1918), 235

[2] B. Carter, "Global structure of the Kerr family of gravitational fields," Phys. Rev. 174 (1968) 1559.

[3] E. G. Kalnins, W. Miller, "Killing tensors and variable separation for Hamilton-Jacobi and Helmholtz equations," SIAM J. Math. Anal., 11 (1980) 1011-1026.

[4] E. G. Kalnins, W. Miller, "Killing Tensors and Nonorthogonal Variable Separation for Hamilton Jacobi Equations," SIAM J. Math. Anal., 12 (1981) 617-629.

[5] S. Benenti, "Intrinsic characterization of the variable separation in the Hamilton Jacobi equation," J. Math. Phys. 38 (1997) 6578-6602.

[6] S. Benenti, C. Chanu, G. Rastelli, "Variable-separation theory for the null Hamilton-Jacobi equation," J. Math. Phys., 46 (2005) 042901.

[7] C. Duval and G. Valent, "Quantum integrability of quadratic Killing tensors," J. Math. Phys. 46 (2005) 053516. arXiv:math-ph/0412059

[8] G. Gibbons, R. Rietdijk and J.W. van Holten, "SUSY in the sky," Nucl. Phys. B404 (1993), 42; arXiv:hep-th/9303112 v1

[9] J.W. van Holten, "Covariant hamiltonian dynamics," Phys. Rev. D75 025027 (2007), arXiv:hep-th/0612216v2 
[10] D. Baleanu, "Geometric duality and third-rank Killing tensors," Grav. Cosmol. 5 (1999) 285

[11] Takahisa Igata, Tatsuhiko Koike, Hideki Ishihara, "Constants of Motion for Constrained Hamiltonian Systems," Phys. Rev. D 83 065027, (2011). arXiv:1005.1815.

[12] G. W. Gibbons, T. Houri, D. Kubiznak and C. M. Warnick, "Some Spacetimes with Higher Rank Killing-Stackel Tensors," Phys. Lett. B 700 (2011) 68, arXiv:1103.5366 [gr-qc]]

[13] G. W. Gibbons and C. Rugina, "Goryachev-Chaplygin, Kovalevskaya, and Brdička-EardleyNappi-Witten pp-waves spacetimes with higher rank Stäckel-Killing tensors," J. Math. Phys. 52 (2011) 122901 arXiv:1107.5987 [gr-qc]].

[14] A. Galajinsky, "Higher rank Killing tensors and Calogero model," Phys. Rev. D 85 (2012) 085002; arXiv:1201.3085 [hep-th]]

[15] M. Visinescu, "Higher order first integrals, Killing tensors and Killing-Maxwell system," J. Phys. Conf. Ser. 343 (2012) 012126.

[16] N. S. Simonović and R. G. Nazmitdinov, "Hidden symmetries of two-electron quantum dots in a magnetic field," Phys. Rev. B67, 041305(R) (2003);

[17] Y. Alhassid, E. A. Hinds and D. Meschede, "Dynamical Symmetries of the Perturbed Hydrogen Atom : the van der Waals Interaction," Phys. Rev. Lett. 59 (1987) 1545; K. Ganesan and M. Lakshmanan, "Comment on "Dynamical Symmetries of the Perturbed Hydrogen Atom : the van der Waals Interaction"”, Phys. Rev. Lett. 629 (1989) 232.

[18] R. Blümel, C. Kappler, W. Quint, and J. Walter, "Chaos and order of laser-cooled ions in a Paul trap," Phys. Rev. A40, 808 (1989). Erratum: Phys. Rev. A 46, 8034 (1992).

[19] P.-M. Zhang, L.-P. Zou, P.A. Horvathy and G.W. Gibbons, "Separability and Dynamical Symmetry of Quantum Dots," Annals of Physics (N.Y.) 341, 94 - 116 (2014) arXiv:1308.3035 [hep-th]].

[20] C. Duval, G. W. Gibbons, P. Horváthy, "Celestial mechanics, conformal structures and gravitational waves", Phys. Rev. D 433907 (1991) hep-th/0512188.

[21] J.W. van Holten, "Gravitational Waves and Black Holes: an Introduction to General Relativity," Fortschr. Phys. 45 (1997), 439 [arXiv:gr-qc/9704043v1].

[22] S. Durand, J. M. Lina and L. Vinet, "Symmetries Of The Massless Dirac Equation In Minkowski Space," Phys. Rev. D 38 (1988) 3837. "Symmetries Of Massless Dirac Equations And Supersymmetric Quantum Mechanics," Lett. Math. Phys. 17 (1989) 289.

[23] M. Cariglia, G. W. Gibbons, J. -W. van Holten, P. A. Horvathy and P. -M. Zhang, "Conformal 
Killing Tensors and covariant Hamiltonian Dynamics," arXiv:1404.3422 [math-ph]. 